\title{
Lock-in thermography versus dye penetration testing and SEM for in vitro adaptation of smart dental restorative materials (giomers) to the walls of the cavity
}

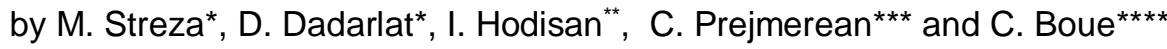 \\ * NIR\&DIMT, 65-103 Donath Street, 400293 Cluj-Napoca, Romania, streza.mihaela@gmail.com \\ ** Iuliu Haţieganu University of Medicine and Pharmacy, Emil Isac Street, 400023 Cluj-Napoca, Romania \\ ioanahodisan@yahoo.com \\ ** *Babes-Bolyai University, Raluca Ripan Chemistry Research Institute, Fantanele Street 400294 Cluj-Napoca, \\ Romania, cristina.prejmerean@gmail.com \\ ****UPMC/ESPCI, LPEM, UMR CNRS 8213,10 rue Vauquelin 75005 Paris, France, christine.boue@espci.fr
}

\begin{abstract}
Lock-in thermography has been used previously for non-destructive testing of materials, but less work has been carried out in medical applications of this technology. Thin vertical cracks located at the interface between tooth and filling are usually difficult to detect by conventional optically stimulated thermography, due to the mainly heat flow generated perpendicular to the surface. The goal of this study is to measure the minimum detectible open crack in cavities restored with classic giomer by lock-in thermography technique, at a given laser intensity that is not harmful for the tooth. In order to check the capabilities of this technique for sealing evaluation and to test its limitations, complementary investigations were carried out by scanning electron microscopy (SEM) and dye penetrant testing. The results obtained by three different methods are also compared.
\end{abstract}

\section{Lock-in thermography for assessment of dental restorations}

The evaluation of dental restoration in a non-invasive way is a very desirable tool for clinical practice. The case of such biological samples is challenging to analyze as the photothermal signals are generally poor. In order to increase the signal to noise ratios, one way would be to increase the energy of the excitation source, that can lead to the exceeding of the dental safety limit. An optical powder density of $2 \mathrm{~W} / \mathrm{cm}^{2}$ can causes an increase in pulp temperature of about $5^{\circ} \mathrm{C}$, that may cause irreversible trauma to the soft tissue located inside the pulp chamber. One possibility would be to reduce the optical power density at the cost of longer measurement time, such as lock-in detection. In lock-in analysis, the signal resolution can be improved by periodically modulating the excitation signal, and correlating and averaging it over many periods of excitation. The amplitude or phase images resulted after the lock-in detection will bear the presence of the crack as a perturbation or phase-shift of thermal wave. Nevertheless, the signals related to the presence of cracks are very weak compared to the excitation background. To provide the defect signature, the second derivative of the amplitude image is used in order to emphasize the useful signal. An image processing algorithm was developed for obtaining a binary image that indicates the gap signature on the surface.

\section{Specimens and experimental procedure}

Giomers represent a new concept in restorative dentistry, based on novel pre-reacted glass technology, where special glass-ionomer fillers are included in the resin matrix. Due to their properties of fluoride release and recharge, as well as their own action mechanism at filling- bone interface, giomers were introduced in the class of smart materials. The experimental series included 9 teeth, extracted from orthodontic and periodontal reasons. Eighteen box-type Class $\mathrm{V}$ standardized cavities were prepared on the facial and oral surfaces of each tooth, with coronal margins in enamel and apical margins in cementum. The preparations were divided randomly into three equal groups and restored with giomer Beautiful (Shofu), in combination with three different adhesive systems: FL BeautiBond adhesive (Shofu), and two new experimental adhesive systems for which the primer contained polyalchenoic acids modified with polymerizable groups as base components. The teeth were thermocycled and immersed in $2 \%$ methyl blue solution for 12 hours. The specimens were then sectioned buccolingually into 3 slices and the resulted sections were examined under a stereo microscope with 20x magnification. Scanning electron microscopy investigations were performed by using a INSPECTS (FEI company) type microscope. The lock-in thermography experimental set up included a heat source (Laser Quantum OPUS with $\lambda=532 \mathrm{~nm}$ and tuneable power, with $0.5 \mathrm{~W}$ maximum laser power), a function generator, and the infrared camera sensitive in the $1.5 \mu \mathrm{m}-5.1 \mu \mathrm{m}$ wavelength range (FLIR 7200 series). An intensity modulated optical stimulation $(\mathrm{f}=1 \mathrm{~Hz})$ is applied near the dental restoration interface. After lock-in procedure, three images are obtained: the magnitude, the phase and the DC image (corresponding to the frequency $f=0$ ). 


\section{Results}

Figure 1 shows the optical image of specimen 1. No trace of dye at the tooth-restoration interface is detected, which means a perfect marginal adaptation.

The images obtained by lock-in thermography are displayed in Figure 2. Figure 2 (a) represents the DC image which is quite disturbed. As the dentine presents numerous dentinal tubules, the laser beam is scattered by the dentine tissue and the excitation spot is expanded on surface. When light heats the interface between dentine and filling, it is partially refracted and partially reflected. Waves reflecting from the interface will generate a greater photon optical absorption process. Consequently, an increased IR signal is observed nearby the edge of the tooth compared to the excitation region (see Figure 2(b)). The resulting enhanced image after applying the image processing algorithm is shown in Figure2 (c). In figure 10 (d) one can observe a binary image consisting of two lines which reveals the edge of the sample in addition to a gap at dentine-filling interface having a length of about $700 \mu \mathrm{m}$ (23pixels).

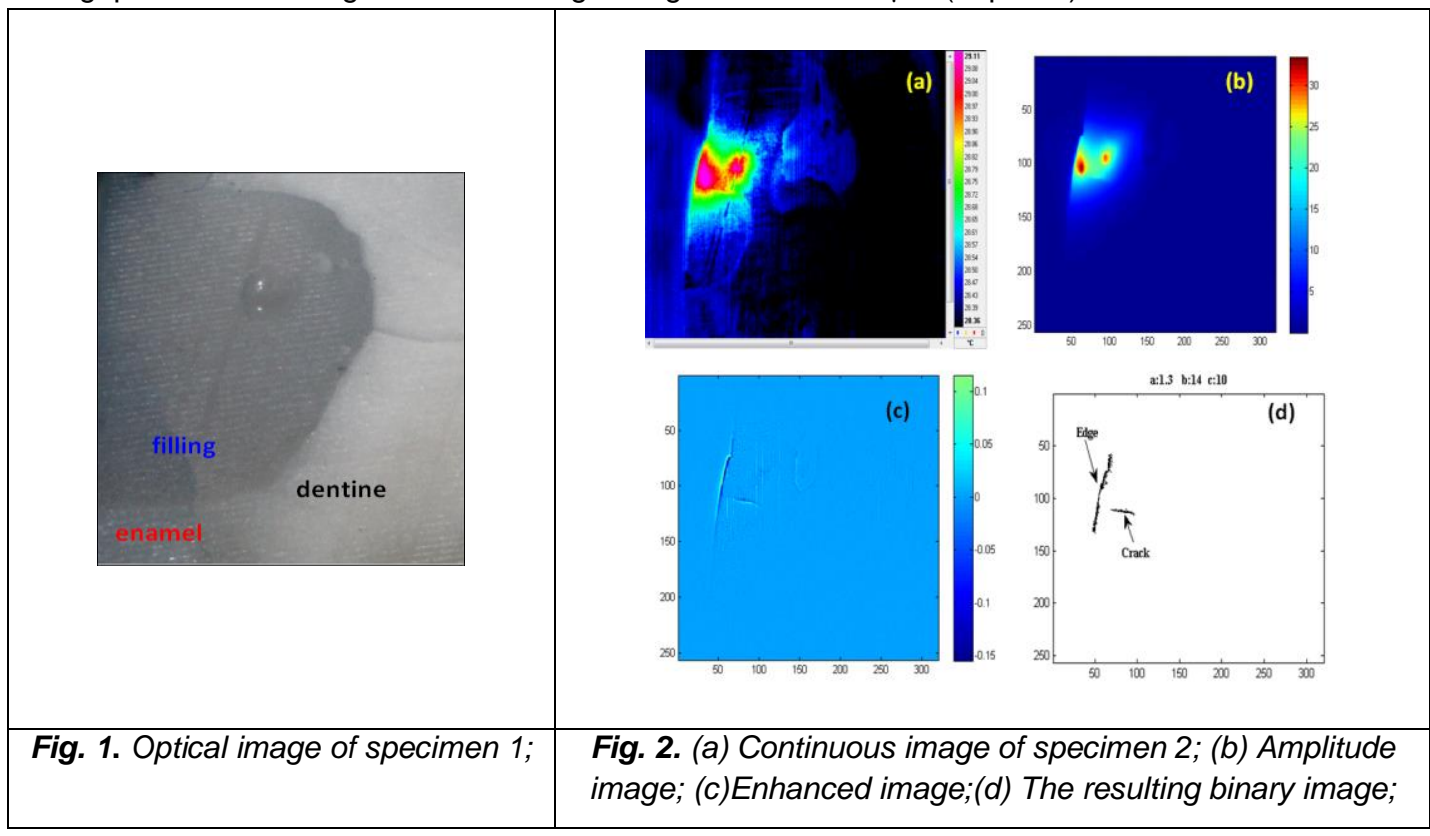

SEM of the restored cavity in the same area where it has been applied lock-in thermography is displayed in Figure 3 ((a)(c)). Figure 3(b) (magnification 306X) shows an internal gap and large secondary electron image along the marked interface (magnification 1000X) indicates a crack having an opening of $5 \mu \mathrm{m}$ at dentine filling interface.

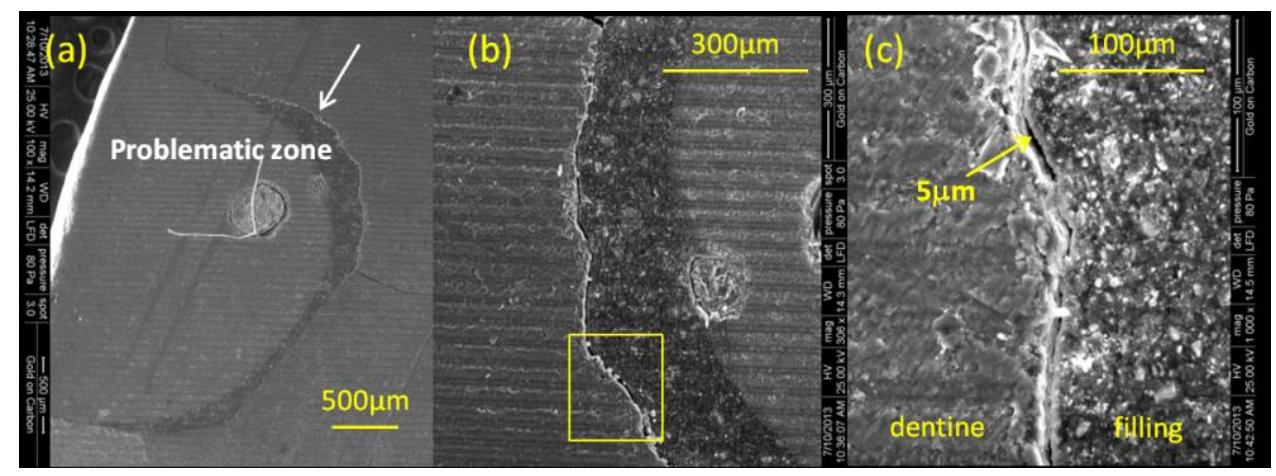

Fig. 3. (a) SEM of the specimen 2; (b) Zoom to the problematic zone (magnification 306X); (c) Zoom to the marked area (magnification 1000X);

These preliminary results show that lock-in thermography and SEM techniques are able to detect flaws located in an internal region of a dental restoration, whereas dye penetrating allows only the detection of marginal gaps. The proposed lock-in procedure leads to a diagnosis of surface-opening cracks having widths less than $1 \mu \mathrm{m}$. 